\title{
SOME PROBLEMS IN POTENTIAL THEORY AND THE NOTION OF HARMONIC ENTROPY
}

\author{
BY BORIS KORENBLUM ${ }^{1}$
}

\begin{abstract}
Blaschke regions are studied for certain classes of subharmonic functions in connection with the notion of harmonic entropy. A complete description of Riesz measures for some of these classes is obtained. A new analytic inequality is established.
\end{abstract}

1. Definitions, notations and two basic problems. $k(r)(0 \leq r<1)$ will always denote a continuous nonnegative function such that $k(|z|)$ is subharmonic in the open unit disc $\mathbf{D}$ (or, equivalently, such that $k(r)$ and $r k^{\prime}(r)$ are nondecreasing).

DEFINITION 1. Let $\mathcal{M} \subset \mathbf{D}$ be a given set, and let $\psi_{\langle k\rangle}(\mathcal{M})$ be the set of all nonnegative harmonic functions $u(z)$ in $\mathbf{D}$ such that $u(z) \geq k(|z|)$ on $\mathcal{M}$. The following quantity will be called the harmonic k-entropy of $\mathcal{M}$ :

$$
\mathcal{E}(\mathcal{M} ; k)=\min \left\{u(0): u \in \mathcal{H}_{\langle k\rangle}(\mathcal{M})\right\} .^{2}
$$

If $\mathcal{H}_{\langle k\rangle}(\mathcal{M})$ is empty, we set $\mathcal{E}(\mathcal{M} ; k)=+\infty$.

DEFINITION 2. $S \mathcal{H}^{\langle\langle k\rangle}$ will denote the class of subharmonic functions $u(z)$ in $\mathbf{D}$ such that

$$
u(z) \leq C_{u} k(|z|) \quad(z \in \mathbf{D}),
$$

where $C_{u}$ is some constant (depending on $u$ ).

DEFINITION 3. $A^{\langle k\rangle}$ will denote the class of analytic functions $f(z)$ in D such that $\log |f(z)| \in S \not{H}\langle k\rangle$.

DEFINITION 4. A region $G \subset \mathrm{D}$ is called a $k$-Blaschke region if either of two equivalent ${ }^{3}$ conditions holds:

(a) for every $u \in S \mathcal{H}_{\langle k\langle}$

$$
b(G ; d \mu)=\int_{G}(1-|z|) d \mu(z)<\infty,
$$

where $d \mu=\Delta u$ is the Riesz measure (i.e. generalized Laplacian) of $u$;

(b) for every $f \in \mathcal{A}^{\langle k\rangle}$

$$
\sum_{z_{\nu} \in G}\left(1-\left|z_{\nu}\right|\right)<\infty
$$

where $\left\{z_{\nu}\right\}$ is the zero set of $f$.

Received by the editors January 28, 1982 and, in revised form, September 3, 1982.

1980 Mathematics Subject Classification. Primary 31A05, 30C15; Secondary 26D15.

${ }^{1}$ Supported by NSF grant MCS80-03413.

${ }^{2}$ The use of that term, borrowed from Information Theory, is suggested by this interpretation: if $u(z)$ is conceived as a "signal" of strength $u(0)$ and $k(|z|)$ as the "noise", then $\mathcal{E}(\mathcal{M} ; k)$ is the strength of the weakest signal that overcomes the noise on $\mathcal{M}$.

${ }^{3}$ The equivalence of (a) and (b) is easily proved. 
DEFINITION 5. (1) $S_{\zeta}$ is the open Stolz angle whose closure is the convex hull of the disk $|z|<1 / \sqrt{2}$ and the point $\zeta \in \partial D$.

(2) For a given closed set $F \subset \partial \mathbf{D}, G_{F}$ is the union of $S_{\zeta}(\zeta \in F)$.

(3) $\mathcal{L}$ is the class of regions $G=\left\{z=r e^{i \theta}: 0 \leq r<f(\theta) \leq 1\right\}$, where $f(\theta)$ is a $2 \pi$-periodic function satisfying the Lipschitz condition $\left|f\left(\theta_{1}\right)-f\left(\theta_{2}\right)\right| \leq$ $\left|\theta_{1}-\theta_{2}\right|$. It is easily seen that every $G_{F}$ is an $\mathcal{L}$-region.

All results announced ${ }^{4}$ below are assoicated with the following two basic problems.

(A) Given $k(r)$, characterize regions of finite $k$-entropy and find estimates of that quantity.

(B) Given $k(r)$, characterize $k$-Blaschke regions and find effective estimates of the integral (1.3) and the sum $\left(1.3^{\prime}\right)$.

The main motivation for (B) is to ultimately obtain a complete description of zero sets for $A^{\langle k\rangle}$-an objective that we are able to realize only for the case of "slowly increasing" $k(r)$. Since the problem of $A^{\langle k\rangle}$-zero sets is essentially a potential-theoretic one, there seems to be no good reason for studying only the special Riesz measures $d \mu=\Delta \log |f(z)|$ determined by the zeros of an $f \in \mathcal{A}^{\langle k\rangle}$, rather than the general Riesz measures for $S \mathcal{K}\langle k\rangle$. In emphasizing the potential-theoretic, rather than complex-analytic, aspect, we also aim at similar multidimensional problems; in fact, some interesting results [4] for the unit ball in $\mathbf{R}^{m}$ have recently been obtained in this circle of ideas (see also $\S 3$ below).

Understanding the structure of $\mathcal{A}^{\langle k\rangle}$-zero sets is also an essential first step towards a satisfactory factorization theory for $A^{\langle k\rangle}$; see [1], where the case $k(r)=|\log (1-r)|$ is treated.

As to (A), this problem is instrumental in solving (B). For slowly increasing $k(r)$ the $k$-entropy of an $\mathcal{L}$-region $G$ can be estimated in terms of the following integral

$$
I(G ; k)=\int_{0}^{2 \pi} k[f(\theta)] d \theta
$$

where $k(1)=k\left(1^{-}\right)(=\infty$, except for the trivial case of a bounded $k(r))$.

In the particular case $k(r)=(1-r)^{-\alpha}(0<\alpha<1)$ our problems lead to a new elementary inequality (3.3).

2. Slowly increasing $k(r)$. In this section an additional condition is imposed on $k(r)$ ( $C$ is some constant):

$$
k\left(1-x^{2}\right) \leq C k(1-x) \quad\left(0<x<\frac{1}{2}\right) .
$$

THEOREM 1. (i) $A G \in \mathcal{L}$ is a $k$-Blaschke region if and only if $I(G ; k)<\infty$.

(ii) There is a constant $\lambda>1$ depending only on $k(r)$ with the property that for every $G \in \mathcal{L}$ there is a $G^{\prime} \in \mathcal{L}, G^{\prime} \supset G$, such that $I\left(G^{\prime}, k\right)<\lambda I(G ; k)$ and

$$
\lambda^{-1} I(G ; k) \leq \mathcal{E}\left(G^{\prime}, k\right)<\lambda I(G ; k) .
$$

\footnotetext{
${ }^{4}$ Detailed proofs will be published elsewhere.
} 
THEOREM 2. The necessary and sufficient condition for a nonnegative Borel measure $d \mu$ in $\mathbf{D}$ to be the Riesz measure of a function $u \in S \psi^{\langle\langle k\rangle}$ is

$$
b\left(G_{F} ; d \mu\right) \leq C I\left(G_{F} ; k\right)
$$

for all finite sets $F \subset \partial \mathbf{D}$ ( $C$ is some constant). In this case (2.3) holds also for all $G \in \mathcal{L}$, but perhaps with a greater constant $C$.

3. Some other results. (1) A Stolz angle is a Blaschke region for $S \mathcal{H}^{\langle k\rangle}$ if and only if

$$
\int_{0}^{1}\left[k(r)(1-r)^{-1}\right]^{1 / 2} d r<\infty .
$$

(See [2].) A similar result for the unit ball in $\mathbf{R}^{m}$ (with $1 / m$ substituted for $1 / 2$ in (3.1)) has recently been obtained by Krzysztof Samotij (written communication).

(2) Consider the region $G=\left\{z \in \mathbf{D}: M\left(1-|z|^{2}\right)|1-z|^{-2}>k(|z|)\right\}$, where $M$ is large enough to ensure that $G \supset S_{1}$. Then (3.1) implies $I(G ; K)<$ $\infty$ and $\mathcal{E}(G ; k)<\infty$.

(3) A recent result by C. N. Linden [3] shows that, under some extra conditions on the regularity of growth of $k,(3.1)$ implies that the above region $G$ is a Blaschke region for $S \mathcal{H}^{\langle\langle k\rangle}$. Similar results describing some "tangential" Blaschke regions for the ball in $\mathbf{R}^{m}$ are given in [4].

(4) In attempting to extend the results of $\S 2$ to wider classes of subharmonic functions, it is natural to consider the particular case $k(r)=(1-r)^{-\alpha}$, where $\alpha$ is fixed, $0<\alpha<1$. In this case the assertion (i) of Theorem 1 still holds, provided the function $r=f(\theta)$, which describes the boundary of $G$, has a finite number of maxima and minima. The proof of this depends on

THEOREM 3. There is a constant $C_{\alpha}$ such that for arbitrary real $x_{0}<x_{1}<$ $\cdots<x_{n}$ satisfying

$$
x_{1}-x_{0} \leq x_{2}-x_{1} \leq \cdots \leq x_{n}-x_{n-1},
$$

and for arbitrary nonnegative $\left\{m_{i}\right\}_{0}^{n}$, the following inequality holds:

$$
\begin{gathered}
\int_{x_{0}}^{x_{n}}\left\{\sum_{0}^{n} m_{i}\left(x-x_{i}\right)^{-2}\right\}^{\alpha /(\alpha+1)} d x \\
\leq C_{\alpha}\left(\sum_{0}^{n} m_{i}\right)^{\alpha /(\alpha+1)}\left\{\sum_{1}^{n}\left(x_{i}-x_{i-1}\right)^{1-\alpha}\right\}^{1 /(\alpha+1)} .
\end{gathered}
$$

Because of the restriction (3.2), which cannot be dropped altogether, our results for this case fall short of a complete description of Riesz measures.

\section{REFERENCES}

1. B. Korenblum, An extension of the Nevanlinna theory, Acta Math. 135 (1975), 187-219.

2. W. K. Hayman and B. Korenblum, A critical growth rate for functions regular in a disk, Michigan Math. J. 27 (1980), 21-30. 
3. C. N. Linden, Regular functions of restricted growth and their zeros in tangential regions, preprint.

4. P. J. Rippon, The boundary behaviour of certain delta-subharmonic functions, preprint.

DEPARTMENT OF MATHEMATICS AND Statistics, State UNIVERSity OF NEW YORK AT ALBANY, ALBANY, NEW YORK 12222 